\title{
MOTIVOS DA NÃO ADESÃO DE CRIANÇAS À CAMPANHA DE VACINAÇÃO CONTRA A INFLUENZA
}

\author{
Josiane Steil Siewert", Dayane Clock², Poliana Giacomin Mergner³, Patrícia Fernandes Albeirice da \\ Rocha $^{4}$, Marceli Diana Helfenstein Albeirice da Rocha ${ }^{5}$, Angela Maria Alvarez ${ }^{6}$
}

Objetivo: conhecer os motivos da não adesão dos pais/responsáveis de crianças à campanha de vacinação contra a influenza. Método: pesquisa quantitativa, tipo descritiva. Critérios de inclusão: pais ou responsáveis de crianças de 6 meses até 4 anos, residentes no município de Joinville. Foram entrevistados 380 responsáveis de crianças entre 6 meses e 4 anos, por meio de questionário semiaberto, em 2016, e realizada análise descritiva dos dados. Resultados: a campanha atingiu a meta, com $89 \%$ das crianças vacinadas. Os motivos da não adesão à campanha foram: medo da reação adversa $(21 ; 51,3 \%)$; informação de que a criança estava gripada $(10 ; 24,3 \%)$; e desconhecimento sobre a Campanha cinco (12,4\%). Conclusão: a enfermagem tem papel fundamental na elaboração, planejamento e execução das campanhas de vacinação. Este estudo evidenciou a necessidade de melhorar as estratégias de educação em saúde referentes à vacinação contra o vírus da influenza e ampliar o acesso na Atenção Primária.

DESCRITORES: Enfermagem; Enfermagem Pediátrica; Programas de imunização; Promoção da saúde; Vacinas contra influenza.

\section{MOTIVES FOR NON-ADHERENCE OF CHILDREN TO THE VACCINATION CAMPAIGN AGAINST INFLUENZA}

Objective: get to know the reasons for non-adherence of parents/responsible caregivers of children to the vaccination campaign against influenza. Method: quantitative and descriptive research. Inclusion criteria: parents or responsible caregivers of children between six months and up to four years of age, living in the city of Joinville. Interviews were held with 380 responsible caregivers of children aged between six months and four years, using a semi-open questionnaire, in 2016, and the descriptive analysis was applied to the data. Results: the campaign reached the target, with $89 \%$ of the children being vaccinated. The reasons for non-adherence to the campaign were: fear of adverse reaction $(21 ; 51.3 \%)$; information that the child had the flue $(10 ; 24.3 \%)$; and not knowing about the campaign $(5 ; 12.4 \%)$. Conclusion: nursing plays a fundamental role in the elaboration, planning and execution of vaccination campaigns. This study evidenced the need to improve the health education strategies concerning vaccination against the influenza virus and to broaden the access to Primary Health Care.

DESCRIPTORS: Nursing; Pediatric Nursing; Immunization programs; Health promotion; Influenza vaccines.

\section{MOTIVOS DE NO ADHESIÓN DE NIÑOS A LA CAMPAÑA DE VACUNACIÓN CONTRA LA INFLUENZA}

Objetivo: Conocer los motivos de no adhesión de padres/responsables de niños a la campaña de vacunación contra la influenza. Método: Investigación cuantitativa, descriptiva. Criterios de inclusión: Padres o responsables de niños de 6 meses a 4 años, residentes en municipio de Joinville. Fueron entrevistados 380 responsables de niños de entre 6 meses y 4 años, utilizándose cuestionario semiabierto en 2016 , realizándose análisis descriptivo de los datos. Resultados: La campaña alcanzó la meta, 89\% de los niños fue vacunado. Los motivos de no adhesión a la campaña fueron: Temor a reacciones adversas $(21 ; 51,3 \%)$; información de que el niño estaba engripado (10; 24,3\%); desconocimiento sobre la Campaña (5; $12,4 \%)$. Conclusión: Enfermería juega un rol fundamental planificando y ejecutando las campañas de vacunación. Este estudio evidenció necesidad de mejorar estrategias educativas en salud respecto de la vacunación contra el virus de la influenza, y de incrementar el acceso a la Atención Primaria. DESCRIPTORES: Enfermería; Enfermería Pediátrica; Programas de Inmunización; Promoción de la Salud; Vacunas contra la Influenza.

\footnotetext{
${ }^{1}$ Enfermeira. Doutoranda em Enfermagem. Docente no Instituto Federal de Santa Catarina (IFSC). Joinville, SC, Brasil.

2 Enfermeira. Doutoranda em Saúde e Meio Ambiente. Docente no Instituto Federal de Santa Catarina (IFSC). Joinville, SC, Brasil.

${ }^{3}$ Enfermeira assistencial no Hospital Materno Infantil Dr Jeser Amarante Farias (HJAF). Joinville, SC, Brasil.

${ }^{4}$ Enfermeira. Mestre em Enfermagem. Docente no Instituto Federal de Santa Catarina (IFSC). Joinville, SC, Brasil.

${ }^{5}$ Enfermeira. Mestre em Saúde Pública. Docente no Instituto Federal de Santa Catarina (IFSC). Joinville, SC, Brasil.

${ }^{6}$ Enfermeira. Doutora em Enfermagem. Docente na Universidade Federal de Santa Catarina (UFSC). Florianópolis, SC, Brasil.
}

Autor Correspondente:

Patrícia Fernandes Albeirice da Rocha.

Instituto Federal de Santa Catarina (IFSC).

R. Pavão, 1377 - 89220-618. Costa e Silva, Joinville, SC, Brasil.

E-mail: patricia.albeirice@ifsc.edu.br.
Recebido: 07/07/2017 Finalizado: 01/06/2018 


\section{INTRODUÇÃO}

A influenza é uma infecção viral aguda do trato respiratório, com distribuição global e elevada transmissibilidade. Seus principias sintomas são: início súbito de febre, calafrios, tremores, dor de cabeça e tosse seca. Em geral, tem evolução autolimitada, de poucos dias. A gripe pode ser causada pelo vírus influenza A, B ou C. Os tipos A e B são os que apresentam maior importância clínica. Os dois tipos sofrem frequentes mutações, razão pela qual são responsáveis pela maioria das epidemias sazonais. No Brasil, o padrão de sazonalidade varia entre as regiões, mas é mais marcado naquelas com estações climáticas bem definidas, ocorrendo com maior frequência em locais de clima temperado, nos meses mais frios ${ }^{(1)}$.

A política pública de vacinação contra a influenza teve início, no Brasil, em 1999, incorporada no Programa Nacional de Imunizações. Por sua vez, a vacinação contra o H1N1 começou em 2010, após a pandemia de 2009. Diferentemente de outras campanhas, a vacinação contra a influenza não tem como objetivo a redução da sua incidência, mas, sim, a redução de internações, complicações e mortes da população ${ }^{(1-2)}$.

O vírus influenza é importante agente causador de infecções respiratórias agudas nas crianças menores de 5 anos de idade, com incidência mais elevada do que na maioria dos grupos etários. Ủm fator agravante é o seu difícil diagnóstico, pois muitas vezes o quadro clínico é inespecífico ${ }^{(3)}$.

Em 2010 houve, no Brasil, 3.184 casos de influenza (H1N1) em menores de 5 anos. No estado de Santa Catarina ocorreram 162 casos, um deles em Joinville ${ }^{(4)}$. Pesquisas realizadas demonstram que crianças menores de 3 meses de idade apresentam risco mais elevado de hospitalização por influenza do que as crianças entre 3 e 12 meses. A maioria das internações foi registrada em crianças saudáveis (75\%), destas, $10 \%$ foram internadas na unidade de tratamento intensivo e $4 \%$ tiveram insuficiência respiratória. Os desafios para a proteção das complicações da influenza dos grupos vulneráveis, entre eles idosos, lactentes e pessoas imunocomprometidas são enormes ${ }^{(5)}$.

Ao analisar os dados da campanha de 2014 no município de Joinville, a meta de vacinação era atingir 131.086 pessoas. Em maio de 2014, segundo os dados fornecidos pelas Unidades de Saúde e Regionais, foram vacinadas 90.662 pessoas, entre elas, crianças de 6 meses até 5 anos, gestantes, puérperas, idosos, trabalhadores da área da saúde e doentes crônicos, alcançando $69,2 \%$ da população-alvo. Foi preciso estender a campanha para alcançar a meta ${ }^{(6)}$.

No ano de 2016, as taxas de vacinação em crianças menores de 5 anos foram: 88,1\% no Brasil; 91,50\% em Santa Catarina; e 83,9\% em Joinville. Embora o município tenha atingido a meta de vacinação, ficou abaixo das médias nacionais e estaduais ${ }^{(4)}$. No início desse ano havia sido constatado um alto número de mortes e internações pelo vírus H1N1 em todo o País, o que mobilizou o Ministério da Saúde e a Sociedade, elevando os esforços para vacinar a população-alvo.

Constatou-se uma intensificação das campanhas de vacinação a partir da pandemia do vírus influenza em 2009, no entanto, é necessário entender quais os motivos que levam as pessoas a não se vacinarem ${ }^{(7)}$. Outro aspecto a considerar é que as pesquisas sobre vacinação envolvem, em sua maioria, os idosos e os profissionais de saúde ${ }^{(2)}$.

Esta pesquisa teve por objetivo conhecer os motivos da não adesão dos pais/responsáveis das crianças à campanha de vacinação contra o vírus A H1N1 em um município da região nordeste de Santa Catarina.

\section{METODOLOGIA}

Trata-se de uma pesquisa quantitativa, do tipo descritiva. Esta pesquisa faz parte de um macroprojeto que teve por objetivo identificar quais os motivos da não adesão da população-alvo à Campanha de Vacinação Contra o Vírus da Influenza, no município de Joinville. Neste artigo, serão apresentados os resultados referentes às crianças. Critério de inclusão: pais ou responsáveis de crianças de 6 meses até 4 anos. Critério de exclusão: pessoas que não fossem residentes do município de Joinville. 
Para determinar o tamanho da amostra, utilizou-se do método baseado na estimativa da proporção populacional, considerando uma população finita. Como as proporções populacionais não eram conhecidas a priori, empregaram-se $p=q=0,5$, grau de confiança de $95 \%$ e margem de erro de $5 \%$. Desta forma, aplicou-se a fórmula ao grupo que se desejava estudar, chegando-se ao tamanho da amostra da população. A população selecionada foi de 34.288 e a amostra, 380. A amostra foi dividida proporcionalmente para a população das nove regionais de saúde, conforme Tabela 1.

Tabela 1 - Amostra por Regional. Joinville, SC, Brasil, 2016

\begin{tabular}{|c|c|c|}
\hline Amostra & $\mathrm{n}$ & $\%$ \\
\hline Pirabeiraba & 16 & 4,2 \\
\hline Vila Nova & 30 & 7,8 \\
\hline Aventureiro & 56 & 14,7 \\
\hline Costa e Silva & 53 & 14 \\
\hline Floresta & 38 & 10 \\
\hline Centro & 34 & 9 \\
\hline Jarivatuba & 53 & 14 \\
\hline Comasa & 47 & 12,3 \\
\hline Fátima & 53 & 14 \\
\hline Total & 380 & 100 \\
\hline
\end{tabular}

Os dados foram coletados em locais públicos e centros de educação infantil (CEI). Foram respeitadas a quantidade de entrevistas por Gerência Regional de Saúde. A coleta de dados foi realizada nos meses de janeiro a maio de 2016, por meio de um questionário semiaberto, cujas variáveis demográficas foram idade, sexo, local de residência e grau de escolaridade. Para esta pesquisa, os dados sobre grau de escolaridade não foram coletados, considerando-se a faixa etária da população-alvo.

As questões fechadas abordaram: a situação vacinal em relação à H1N1(sim/não); local de vacinação (rede pública/rede privada/outros); como o entrevistado avaliava a divulgação da campanha de vacinação (bem divulgada/pouco divulgada); como a pessoa ficou sabendo da campanha (televisão/rádio/agente comunitário de saúde/outros); e o que poderia ser feito para melhorar a campanha nos próximos anos (esclarecer melhor a importância da vacinação/ampliar a divulgação da campanha/estender o horário de atendimento das unidades básicas de saúde/oferecer mais de um dia "D" de vacinação/outros).

A questão sobre o motivo de não se vacinar era aberta, permitindo, portanto, que o participante pudesse opinar livremente, sem interferências do pesquisador. Para a análise, os dados foram tabulados em planilha eletrônica e analisados por estatística descritiva. Os dados das questões abertas foram agrupados por proximidade dos temas, gerando as informações. O estudo respeitou as exigências formais contidas nas normas nacionais e internacionais regulamentadoras de pesquisas envolvendo seres humanos sob o parecer de número 991.967.

\section{RESUlTADOS}

Participaram desta pesquisa 380 responsáveis de crianças entre 6 meses e 4 anos. O local de residência foi proporcional ao cálculo amostral. Na Tabela 2, são apresentadas as características demográficas dos participantes da pesquisa e a distribuição dos vacinados e não vacinados. 
Tabela 2 - Características demográficas das 380 responsáveis de crianças participantes da pesquisa e distribuição dos vacinados e não vacinados. Joinville, SC, Brasil, 2016

\begin{tabular}{lccc}
\hline Características demográficas & $\mathbf{n}(\%)$ & Vacinados & Não vacinados \\
\hline Sexo & & $\mathbf{n}(\%)$ & $\mathbf{n}(\%)$ \\
Feminino & $207(54,5)$ & $186(89)$ & $21(11)$ \\
Masculino & $173(45,5)$ & $153(88,4)$ & $20(11,6)$ \\
Faixa etária & & & \\
6 a 11 meses & $52(14)$ & $50(96)$ & $2(4)$ \\
1 ano & $69(18)$ & $64(93)$ & $5(7)$ \\
2 anos & $71(19)$ & $64(90)$ & $7(10)$ \\
3 anos & $84(22)$ & $69(82)$ & $15(18)$ \\
4 anos & $104(27)$ & $92(88)$ & $12(12)$ \\
\hline
\end{tabular}

Do total dos entrevistados, apenas $21(11 \%)$ não levaram seus filhos para receber a vacina, com distribuição semelhante entre os sexos. Com relação à gerência regional de saúde, apenas uma unidade não atingiu a meta de $80 \%$, chegando muito próximo (79\%). Na rede pública, foram vacinadas 319 $(84,1 \%)$ crianças e na privada, $61(15,9 \%)$.

A campanha de vacinação foi relatada como bem divulgada por $260(70 \%)$ dos entrevistados. A televisão, apontada por 254 (67\%) dos entrevistados, foi a principal forma de divulgação da campanha, seguida por: agente comunitário de saúde $(57 ; 15,0 \%) ; 49$ (13\%) por outros meios de divulgação; 15 $(4 \%)$ por meio do rádio; e cinco (1\%) relataram desconhecer a campanha de vacinação. A seguir, são apresentados os dados sobre os motivos da não adesão à campanha de vacinação (Tabela 3).

Tabela 3 - Motivos da não adesão à Campanha de Vacinação. Joinville, SC, Brasil, 2016

\begin{tabular}{lcc}
\hline Motivos & $\mathbf{N}$ & \% \\
\hline Medo da reação adversa & 21 & 51,3 \\
Criança estava gripada & 10 & 24,3 \\
Não sabia da campanha & 5 & 12,1 \\
Criança era alérgica & 3 & 7,3 \\
Pediatra não autorizou que fizesse a vacina & 1 & 2,5 \\
Vacina em falta na Unidade de Saúde & 1 & 2,5 \\
\hline Total & 41 & 100 \\
\hline
\end{tabular}

Sobre o que poderia ser feito para melhorar a adesão à campanha de vacinação, $108(28,5 \%)$ dos entrevistados disseram que seria necessário esclarecer melhor a importância da vacinação, 86 (22,7\%) ampliar a divulgação da campanha, 79 (20,7\%) estender o horário de atendimento nas unidades de saúde, e 71 (18,6\%) oferecer mais de um dia "D" de vacinação.

A população que foi comunicada pelo Agente Comunitário de Saúde, conforme a regional de saúde, constituiu-se em 17 (32\%) da regional do Aventureiro, cinco (31,2\%) da regional Pirabeiraba, 14 (26,4\%) da regional Costa e Silva, quatro $(13 \%)$ da regional Vila Nova, quatro $(10,5 \%)$ da regional Floresta, cinco $(9 \%)$ da regional Aventureiro, três $(8,8 \%)$ da regional Centro, dois $(4,2 \%)$ da regional Comasa e um $(1,8 \%)$ da regional Fátima. 
A faixa etária predominante neste estudo foi composta de crianças entre 3 e 4 anos (49\%). Isto se justifica pelo fato de a coleta de dados ter ocorrido em Centros de Educação Infantil e nas ruas da cidade, ambientes de menor circulação de crianças até 2 anos.

A maioria dos entrevistados avaliou que a Campanha foi bem divulgada. Como forma de propaganda, a televisão atingiu a maioria da amostra, e, somando-se ao que foi realizado via rádio, as mídias perfizaram 270 (71\%) entrevistados. A mídia é, de fato, o veículo informativo mais abrangente nesta pesquisa, pelo amplo alcance junto à população. Conforme dados da Pesquisa Brasileira de Mídia, 95\% dos entrevistados afirmaram possuir televisão, e 48\% ter acesso à internet ${ }^{(8)}$.

Pesquisa sobre a campanha de vacinação contra o Papiloma Vírus Humano apontou que 41,7\% das pessoas souberam da campanha pela mídia, corroborando o fato de a televisão, rádio e internet serem os meios de comunicação mais utilizados pelos brasileiros como forma de auxiliar na divulgação de campanhas de promoção à saúde ${ }^{(9)}$.

Porém, é necessário cautela, pois, embora seja forte aliada na divulgação de campanhas, a mídia por si só não intervém, de fato, na educação em saúde das pessoas, pois possui um caráter passivo de informação. Muitas vezes, as mensagens transmitidas pela mídia não são suficientes ou adequadas para que as pessoas se empoderem sobre as medidas de prevenção, pois podem ocorrer equívocos pelos próprios meios de informação ou por dificuldade de interpretação da população(9).

De acordo com os dados coletados, nota-se que a divulgação por meio dos agentes comunitários de saúde foi pouco efetiva e precisa ser aprimorada. Eles têm uma função muito importante na comunicação, pois são membros da equipe que atua na comunidade, o que permite a criação de vínculos e acolhimento mais facilmente, favorecendo assim o contato direto com a equipe ${ }^{(10)}$.

Os agentes comunitários são trabalhadores do Sistema Único de Saúde, com grande importância na coleta de dados e informações que facilitam o acesso da população à saúde. Também possuem papel preponderante nas orientações e divulgação de campanhas e na promoção da educação em saúde. No entanto, o município de Joinville conta com apenas 76,65\% de cobertura de agentes comunitários de saúde. Portanto, muitas áreas não são atendidas por esses trabalhadores ${ }^{(11)}$.

Verificou-se que, dos 380 entrevistados, 186 (89\%) aderiram à campanha e realizaram a vacinação contra o vírus $\mathrm{H} 1 \mathrm{~N} 1$, atingindo e superando a meta mínima de 80\%. Esse resultado está de acordo com os encontrados no Departamento de informática do Sistema Único de Saúde (DATASUS), cujos dados apontaram adesão à Campanha por parte de 83,93\% da população, no município de Joinville ${ }^{(4)}$.

Pesquisas realizadas sobre a cobertura vacinal no Brasil mostraram que as metas de vacinação contra o H1N1 foram superadas. Na campanha de 2010, os grupos com coberturas superiores às estimativas foram: os portadores de doenças crônicas (131,9\%); a população de 6 meses a menos de 2 anos de idade $(127,93 \%)$; e os profissionais de saúde (120\%). O êxito da Campanha repetiu-se em $2011^{(2,12)}$.

A faixa etária que mais apresentou crianças não vacinadas foi a de 3 a 4 anos. Esse dado pode estar relacionado ao número maior de reações adversas na primeira dose da vacina, o que acabou influenciando os pais e/ou responsáveis da criança a não aderirem. No mesmo sentido, outro estudo ${ }^{(13)}$ apontou o medo da reação adversa como o principal motivo da não adesão à vacinação.

No ano de 2016 foram notificados, aproximadamente, 1.870 óbitos decorrentes da infecção por H1 N1 no País, no final do mês de março já haviam sido notificadas 102 mortes, das quais 3,5\% eram de crianças menores de 2 anos. Em setembro, 10\% das mortes ocorreram em crianças menores de 5 anos ${ }^{(4)}$. Neste estudo, para 21 (51,3\% das respostas) dos entrevistados, o medo da reação adversa foi maior do que o risco do contágio de uma doença potencialmente letal. Os outros motivos que levaram os pais a não vacinarem seus filhos estavam relacionados à condição clínica da criança, problemas técnicos ou desconhecimento da campanha. 
Entre as estratégias adotadas pelo governo federal para aumentar a adesão às campanhas de vacinação, destacam-se a implementação de ações para o controle e eliminação de doenças, a multivacinação, o dia D de vacinação e a ampliação da população-alvo para vacinação(14).

O terceiro motivo que levou cinco $(12,1 \%)$ pais a não vacinarem seus filhos está relacionado à falta de conhecimento sobre a Campanha de Vacinação. Mesmo com as diversas estratégias de divulgação, há uma parcela da população que não é alcançada. É necessário identificar quem são essas pessoas e planejar ações que ampliem a divulgação da campanha. Reforça-se, aqui, a importância fundamental da atuação dos Agentes Comunitários de Saúde, pois eles podem identificar quem não teve acesso à informação.

Além de prevenir as dificuldades advindas de morte ou doença grave ao núcleo familiar, as campanhas de vacinação auxiliam no controle de gastos com a saúde. Pesquisa realizada em um hospital universitário concluiu que o custo médio por paciente internado foi de $R \$ 21.141,42$, sendo gastos, por dia, $R \$$ $1.496,74^{(15)}$. Na Coreia do Sul, os custos da pandemia de H1N1 de 2009 foram de US\$1,09 bilhões. Os custos médicos diretos foram de US\$322,6 milhões, e os custos diretos não médicos foram de US\$ 105,4 milhões. Os custos indiretos totalizaram US\$ 662,5 milhões ${ }^{(16)}$.

Os dados desta pesquisa sobre o que poderia ser feito para melhorar a campanha apontam melhorias no acesso (mediante a extensão do horário de atendimento e da necessidade de mais de um dia " $\mathrm{D}^{\text {" de }}$ vacinação), esclarecimentos sobre a importância da vacinação e ampliação da divulgação. Percebe-se uma aproximação entre algumas estratégias realizadas pelo governo e outras citadas pela população. No entanto, o que mais chama atenção é a questão do acesso. O acesso, neste caso específico, pode estar relacionado ao horário de trabalho dos pais, inviabilizando o atendimento nos Postos de Saúde, devido ao horário de funcionamento das unidades de saúde.

Outro fator que contribui para a baixa adesão é a falta de conhecimento dos responsáveis sobre a importância da vacinação. Acredita-se que condutas de promoção e proteção entre os profissionais da saúde e a família possam aumentar a adesão e aumentar os índices de vacinação do Programa Nacional de Imunização(17).

Na presente pesquisa, uma das crianças não pôde ser vacinada devido a uma contraindicação médica. Um dos motivos da não adesão às vacinas está relacionado com a resistência das famílias, por causa de contraindicações incorretas ${ }^{(18)}$. Segundo o autor, isso pode ocorrer por causa de informações repassadas erroneamente pelos profissionais. É importante proceder-se a uma investigação quando a família relata que o médico da criança contraindicou a vacina, para avaliar se essa contraindicação é pertinente, e, caso não seja, deve promover-se um treinamento ou reciclagem dos profissionais.

Outros motivos encontrados na literatura da não vacinação das crianças foram: falta de tempo $(32,3 \%)$, falta de vacina no posto $(27,5 \%)$, ausência de comprovante de vacinação $(16,5 \%)$ e dificuldade de acesso ao posto $(10,7 \%)^{(19)}$.

Esses resultados reforçam a necessidade de intervenções educativas na população, para prover informação adequada sobre a prevenção do vírus influenza e sobre a vacinação. Isso significa não apenas transmitir informações científicas sobre a doença, mas fazê-lo de acordo com a capacidade das pessoas para processarem tais informações. Esse objetivo pode ser alcançado inserindo a população de modo efetivo na Atenção Primária, fortalecendo-a no seu objetivo de promoção da saúde ${ }^{(18)}$.

A cobertura vacinal tem êxito quando há um processo integrado. Além de atuar no desenvolvimento e crescimento da criança, a educação por parte dos profissionais deve prover entendimento às famílias acerca das ações de saúde em alguns determinantes importantes, como, por exemplo, a conjuntura política e social, lançando mão da promoção e prevenção da saúde, com a finalidade de troca de conhecimentos. Segundo um estudo realizado, a não adesão está relacionada com fatores multifatoriais, e um dos motivos é o comportamento de negação associado ao preconceito e a fatores relacionados à vulnerabilidade social ${ }^{(17)}$.

Embora este estudo contribua para o conhecimento sobre os motivos da não adesão à campanha de vacinação contra o vírus H1N1 dos pais e responsáveis das crianças, é fundamental explorar ainda como eles obtêm as informações sobre as vacinas. É importante destacar que a pesquisa foi realizada no contexto de um município da região sul do País. Os motivos podem variar devido a questões culturais em 
outros contextos e momentos históricos. Por exemplo, observou-se grande procura pela vacina da febre amarela, devido ao surto que assolou o País recentemente. No entanto, assim que este foi controlado, a procura voltou ao normal. O mesmo fenômeno pôde ser observado em momentos nos quais ocorreram surtos de mortes pelas formas mais graves de gripes, sem, no entanto, ser uma preocupação constate da população.

\section{CONCLUSÃO}

Atualmente, no processo de tomadas de decisão em saúde, é importante que os profissionais e gestores conheçam não apenas os benefícios clínicos das intervenções, mas também realizem análise de custo-benefício, para melhor aplicação dos recursos. Comparando o custo médio da vacina com os custos de internações hospitalares, percebe-se que a prevenção é a melhor opção, tanto financeira como globalmente, pois as consequências das internações hospitalares têm um maior impacto na vida pessoal, bem como na sociedade.

O principal motivo de os pais não terem vacinado as crianças foi o medo da reação adversa. Esse temor coletivo está intimamente relacionado a um imaginário segundo o qual a vacina pode causar uma sequela, risco que não justificaria a prevenção de uma simples gripe. Entre as medidas necessárias para melhorar a cobertura vacinal, pode-se citar: intensificação das informações na Atenção Básica; realização de campanhas em centros de educação infantil e escolas; e, principalmente, fortalecimento do trabalho dos agentes comunitários de saúde.

\section{- REFERÊNCIAS}

1. Ministério da Saúde (BR). Secretaria de Vigilância em Saúde. Departamento de Vigilância Epidemiológica. Guia de Vigilância em Saúde. [Internet] Brasília: Ministério da Saúde; 2016 [acesso em 28 ago 2017]. Disponível: http://www. rio.rj.gov.br/dlstatic/10112/6385405/4170293/GUIADEVS2016.pdf.

2. Luna EJA, Gattas VL, Campos SRSLC. Efetividade da estratégia brasileira de vacinação contra influenza: uma revisão sistemática. Epidemiol. Serv. Saúde. [Internet] 2014;23(3) [acesso em 28 ago 2017]. Disponível: http://dx.doi. org/10.5123/S1679-49742014000300020.

3. Scotta MC. Influenza em pediatria. Bol Cient Pediatr. [Internet] 2013;02(2) [acesso em 28 ago 2017]. Disponível: http://www.sprs.com.br/sprs2013/bancoimg/131210133757bcped_02_03.pdf.

4. Ministério da Saúde (BR). Sistema de Informação do Programa Nacional de Imunizações. Campanha Nacional de Vacinação contra a Gripe 2016. [Internet] Brasília: Ministério da Saúde; 2016 [acesso em 14 ago 2016]. Disponível: http://sipni.datasus.gov.br/si-pni-web/faces/relatorio/consolidado/coberturaVacinalCampanhalnfluenza.jsf.

5. Chaves SS, Perez A, Farley MM, Miller L, Schaffner W, Lindegre ML, et al. The Burden of Influenza Hospitalizations in Infants from 2003- 2012, United States. Pediatr. Infect. Dis. J. [Internet] 2014;33(9) [acesso em 28 ago 2017]. Disponível: http://dx.doi.org/10.1097/INF.0000000000000321.

6. Secretaria Municipal de Saúde de Joinville/ Gerencia da Unidade de Atenção Básica/ Área do Cadastramento, Joinville -2014 .

7. Monteiro CN, Gianini RJ, Goldbaum M, Cesar Chester LG, Barros MBA. Cobertura de serviços públicos de saúde para gastos com medicamentos e vacinas na população com diabetes mellitus. Ciênc. saúde coletiva. [Internet] 2015;20(2) [acesso em 28 ago 2017]. Disponível: http://dx.doi.org/10.1590/1413-81232015202.02112014.

8. Presidência da República (BR). Secretaria de Comunicação Social. Pesquisa brasileira de mídia 2015: hábitos de consumo de mídia pela população brasileira. [Internet] Brasília: Secom; 2014 [acesso em 28 ago 2017]. Disponível: http://www.fndc.org.br/publicacoes/pesquisas-e-relatorios/pesquisa-brasileira-de-midia-2014-habitos-de-consumo-demidia-pela-populacao-brasileira/. 
9. Osis MJD, Duarte GA, de Sousa MH. Conhecimento e atitude de usuários do SUS sobre o HPV e as vacinas disponíveis no Brasil. Rev. Saúde Pública. [Internet] 2014;48(1) [acesso em 28 ago 2017]. Disponível: http://dx.doi.org/10.1590/ S0034-8910.2014048005026.

10. Ministério da Saúde (BR). Secretaria de Atenção à Saúde. Departamento de Atenção Básica. Política Nacional de Atenção Básica (PNAB). [Internet] Brasília: Ministério da Saúde; 2012 [acesso em 28 ago 2017]. Disponível: http://189.28.128.100/dab/docs/publicacoes/geral/pnab.pdf.

11. Secretaria Municipal de Saúde de Joinville. Gerencia da Unidade de Atenção Básica/ Área do Cadastramento, Joinville -2014 .

12. Domingues CMAS, Teixeira AMS. Coberturas vacinais e doenças imunopreviníveis no Brasil no período de 1982 2012: avanços e desafios do Programa Nacional de Imunizações. Epidemiol. Serv. Saúde. [Internet] 2013;22(1) [acesso em 28 ago 2017]. Disponível: http://dx.doi.org/10.5123/S1679-49742013000100002.

13. de Andrade GN, Pimenta AM, Silva DA, Madeira AMF. Eventos adversos pós-vacinação contra influência pandêmica A (H1N1) 2009 em crianças. Cad. Saúde Pública. [Internet] 2012;28(9) [acesso em 28 ago 2017]. Disponível: http:// dx.doi.org/10.1590/S0102-311X2012000900010.

14. Domingues CMAS, Teixeira AMS, Carvalho SMD. National immunization program: vaccination, compliance and pharmacovigilance. Rev. Inst. Med. Trop. S. Paulo. [Internet] 2012;54(Suppl 18) [acesso em 28 ago 2017]. Disponível: http://dx.doi.org/10.1590/S0036-46652012000700009.

15. Silva CS, Haddad MCL, Silva LGC. Custo da internação de pacientes com gripe A (H1N1) em hospital universitário público. Cienc. Cuid. Saude. [Internet] 2012;11(3) [acesso em 28 ago 2017]. Disponível: http://dx.doi.org/10.4025/ cienccuidsaude.v11i3.13729.

16. Kim Y, Yoon S, Oh Y. The economic burden of 2009 pandemic H1N1 influenza in Korea. Scand. J. Infect. Dis. [Internet] 2013;45(5) [acesso em 28 ago 2017]. Disponível: https://doi.org/10.3109/00365548.2012.749423.

17. Sherlock MSM, Cardoso MVLML, Lélis ALPA, Lopes MMCO, Pereira MLD. Motivos da não adesão das mães ao esquema vacinal de filhos expostos ao HIV. Rev. Rene. [Internet] 2013;14(2) [acesso em 28 ago 2017]. Disponível: http://www.periodicos.ufc.br/rene/article/view/3389/2625.

18. Sorensen K, Van Den Broucke S, Fullam J, Doyle G, Pelikan J, Slonska Z, et al. Health literacy and public health: a systematic review and integration of definitions and models. BMC Public Health. [Internet] 2012;12(8) [acesso em 28 ago 2017]. Disponível: https://bmcpublichealth.biomedcentral.com/track/pdf/10.1186/1471-2458-1280 ? site=bmcpublichealth.biomedcentral.com.

19. Teixeira AMS, Domingues CMAS. Monitoramento rápido de coberturas vacinais pós campanhas de vacinação no Brasil: 2008, 2011 e 2012. Epidemiol. Serv. Saúde. [Internet] 2013;22(4) [acesso em 28 ago 2017]. Disponível: http:// dx.doi.org/10.5123/S1679-49742013000400003. 